A. Pramesh Rao, G. Swarup and Gopal-Krishna, eds.

\title{
Wide Field Imaging at Low Frequencies
}

\author{
R.J. Sault \\ Australia Telescope National Facility, P.O. Box 76, EPPING, N.S.W., \\ 1710, Australia
}

\begin{abstract}
Despite seemingly being poles apart, the two techniques of mosaicing and "polyhedron imaging" in radio interferometer do share some common aspects. This note shows some of these similarities. In particular, a simple method for producing geometrically correct images of large fields is described.
\end{abstract}

\section{Mosaicing and polyhedron imaging}

We can think of the techniques of "mosaicing" and "polyhedron imaging" as being two extremes in interferometric wide field imaging.

Mosaicing is needed when the primary beam response is too small compared to the source we are interested in, and so we require many pointings to synthesis a complete field. Interestingly mosaicing should not be viewed as simply a cutand-paste merging of many pointings together. As Cornwell (1988) shows, by jointly processing all the pointings of a mosaic, a fundamentally improved result is possible. In particular, Cornwell notes that mosaicing is a generalization of a technique described by Ekers \& Rots (1979) for estimating short spacings using an interferometer. Although mosaicing is normally thought of as a technique for use at high frequencies (where the primary beam size is small), it is equally applicable at low frequencies for very large objects (e.g. Galactic non-thermal or HI emission).

On the other hand, polyhedron imaging is a technique developed to cope with the low frequency problem where the primary beam is too large (Cornwell \& Perley 1992). As a consequence of the large field, there are many confusing sources that the interferometer is sensitive to, and these sources (or more particularly their sidelobes) need to be correctly accounted for to produce a high quality image. However there is a problem for arrays which do not produce coplanar $u-v-w$ coverage. When this is the case, a distortion arises in regions of an image which are a large distance from the phase centre. Arrays that produce coplanar $u-v-w$ coverage (such as east-west arrays or snapshots from physically coplanar arrays) do not suffer this problem. As an example of the 'evils' of not properly accounting for the non-coplanar baseline effect, Fig. 1 shows the response of a point source $5^{\circ}$ from the phase centre in a simulated VLA observation. Apart from being smeared, the response is also shifted from its correct position.

This "non-coplanar baselines" problem has been known for many years. Indeed Clark (1973) describes it well, and states that to solve it "a simple mosaic 

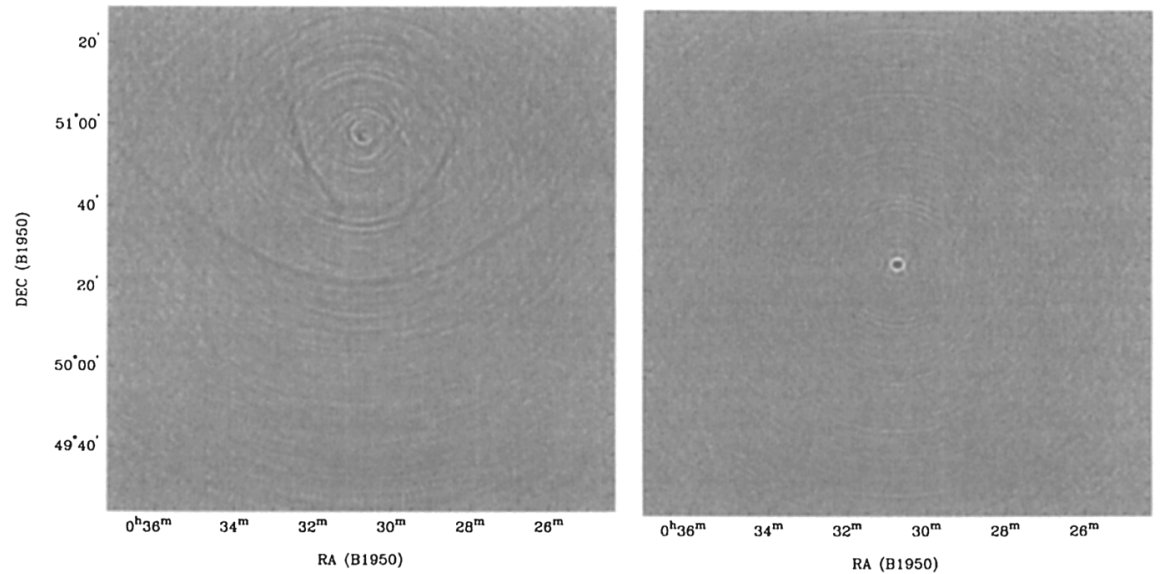

Figure 1. An example of the non-coplanar baseline effect on a point source $5^{\circ}$ from the phase centre in a simulated VLA observation. Left panel shows processing with no correction for the non-coplanar baseline effect, whereas the right panel shows correct handling of the effect.

of fields ... is an attractive way to do things". That is, Clark was suggesting that a primary beam could be split into a number of small fields (or facets), each of which could then be correctly imaged. The 'correct imaging' consists of appropriate re-phasing of the visibility data and applying a transformation to the $u-v$ coordinate. This is the heart of the polyhedron imaging idea.

\section{Similarities and differences}

It is interesting that Clark used the word "mosaic" to describe a remedy for the non-coplanar baseline problem, given that this term is now used for a quite different technique. But it does underline that mosaicing and polyhedron imaging do share a number of similarities: they break the sky into a number of small facets (sub-fields or pointings), they image these individually, and then stitch the facets back together. In both techniques, the stitching of the facets and the deconvolution process are intimately coupled. Also both techniques need to handle a shift-variant point-spread function, as the point-spread function varies from facet to facet.

There are some important differences between the two as well. From an algorithmic point of view, the biggest difference is that the point-spread function for a mosaicing observation is finite in size (or at least it is generally approximated as such). The mosaic point-spread function is no larger than the extent of the primary beam response (or at least our model of this response). This is usually much smaller than the field being synthesized. On the other hand, the non-coplanar baseline effect exists largely because the primary beam size is so 


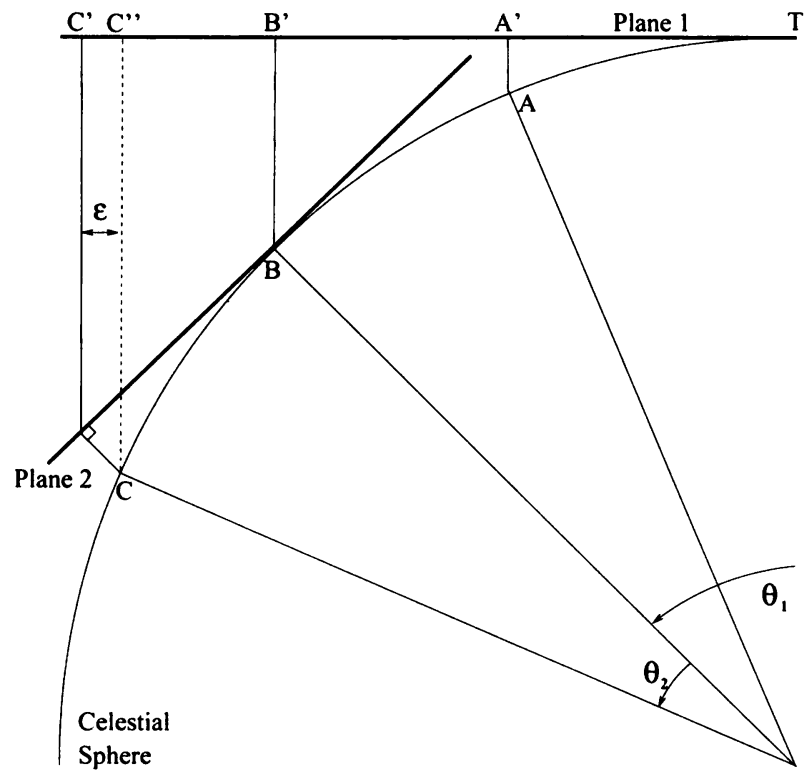

Figure 2. Geometry of various projections involved in combining facets with different tangent planes.

large: the response of a point source typically will extend right across the region being synthesized.

\section{Large field geometry}

The main contribution of this paper is to present a way to produce an image of a large field with a uniform projection geometry. This was described by Sault et al. (1996) in the context of mosaicing, but it is just as relevant to polyhedron imaging. The basic aim of the approach is to form all facets of the final image with the same projection geometry, and in a way so that the pixels of all facets lie on the one global pixel grid (i.e. no regridding or interpolation in the image plane is needed).

Only the case of a non-coplanar observation is considered (coplanar observations are much easier), with the process depicted in Fig. 2. To image each facet, the data first need to be phased up to the centre of that facet, and the $u-v$ coordinates also need to be computed with respect to the facet centre. Imaging these data would result in an image with the so-called "sine" projection geometry, with the tangent point at the centre of each facet. This is not what we desire, as each facet will have somewhat different geometry, as there tangent points are different. For example plane 1 and plane 2 in Fig. 2 have quite different geometries corresponding to tangent points $T$ and $B$. The trick used here to gain a uniform geometry is to re-project plane 2 onto plane 1 . 
The operations required to do this simply involve a matrix transformation of the $(u, v)$ coordinates of the data for plane 2 facet (Sault et al. 1996 give the detailed formulas). To get the pixel grids to align, a shift of at most $\pm \frac{1}{2}$ pixel is also needed - this can be done by way of re-phasing the visibility data. Using this double projection, as depicted in Fig. 2, point $B$ gets re-projected to point $B^{\prime}$, as is desired. This technique is a small field approximation, however, and we can see that point $C$ gets projected to $C^{\prime}$, whereas it should be projected to $C^{\prime \prime}$. This projection error (the distance between $C^{\prime \prime}$ and $C^{\prime}$ ) is

$$
\begin{aligned}
\epsilon & =\sin \theta_{1}\left(1-\cos \theta_{2}\right) \\
& \approx \frac{1}{2} \theta_{1} \theta_{2}^{2} .
\end{aligned}
$$

Note that this error is no worse than the "non-coplanar baseline" distortion present in a given facet (see Perley 1999). If the non-coplanar baseline distortion is tolerable in a given facet, then so should the error in the above projection.

\section{Conclusion}

This geometric approach for polyhedron imaging needs to be compared with the approach of Cornwell \& Perley (1992). Cornwell \& Perley make their facets with different projection geometries, and then only as a final step (after deconvolution) are these facets interpolated onto a common grid. From an algorithmic and computational point of view, the geometric approach advocated here shares many of the strengths and weaknesses of the Cornwell \& Perley approach. The biggest advantage of the current technique should be in the handling of the edges of facets, where facets abut. When all the facets are made with the same geometry and on the same grid, it is easier to jointly handle these edges. Indeed the different facets could be feathered together. The result should be less artifacts at these edges.

\section{References}

Clark, B.G., 1973, VLA Scientific Report 107, NRAO, Socorro

Cornwell, T.J., 1988, A\&A, 202, 316

Cornwell, T.J., Perley, R.A., 1992, A\&A, 261, 353

Ekers, R.D., Rots, A.H., 1979, in Proc. IAU Col. 49, Image Formation from Coherence Functions in Astronomy, ed. C. van Schooneveld, D. Reidel, p. 61

Perley, R.A., 1999, in Synthesis Imaging in Astronomy, ed. G.B. Taylor, C.L. Carilli, R.A. Perley, ASP Conf. Series, 180, 383

Sault, R.J., Staveley-Smith, L., Brouw, W.N., 1996, A\&AS, 120, 375 